\title{
Assessment of the fluid dynamic performance of a vitreous cutter
}

Alessandro Stocchino ${ }^{1}$, Rodolfo Repetto ${ }^{1}$, Mario Romano ${ }^{2}$

${ }^{1}$ Department of Civil, Chemical and Environmental Engineering, University of Genoa, Italy; ${ }^{2}$ Department of Biomedical Sciences, Humanitas University, Milan, Italy

\section{Abstract}

Vitreous cutters are surgical devices used during vitrectomy to remove the vitreous humor from the eye and replace it with tamponade fluids. The aim of the present work is to assess the performance of the EVA Phaco-vitrectomy System vitreous cutter (Dutch Ophthalmic Research Center (DORC) BV; Zuidland, The Netherlands) used with different needle sizes and blade shapes. The analysis is based on laboratory measurements of fluid flow performed using the particle image velocimetry (PIV) technique.

Keywords: particle image velocimetry (PIV), vitrectomy, vitreous cutter

\section{Introduction}

Vitreous cutters are surgical devices used during vitrectomy to remove the vitreous humor from the eye and replace it with tamponade fluids. A lot of attention has been devoted in recent years to optimize the shape of cutter needles with the aim of improving removal efficacy without generating excessive mechanical stress on the retina. The amount of mechanical stress on the retina during the vitrectomy procedure depends on the fluid flow generated during vitreous aspiration. Recently, Rossi et al. performed PIV measurements of fluid flow around the tip of various cutter blades and proposed a way of assessing the performance of the vitreous cutter. ${ }^{1}$ In particular, they propose to evaluate the performance of the device making use of a diagram (see Fig. 8 of their paper), ${ }^{1}$ in which they report the volumetric flux pumped by the cutter on the $y$-axis and the time derivative of the local fluid velocity,

Correspondence: Alessandro Stocchino, Dipartimento di Ingegneria Civile, Chimica e Ambientale, via Montallegro, 1, 16145 Genova, Italy.

E-mail: alessandro.stocchino@unige.it 
$\partial \mathbf{u} / \partial t \equiv \mathbf{u}$ (averaged over a certain spatial domain), on the $\mathbf{x}$-axis. The rationale about the diagram is that, if a cutter is able to produce large fluxes, it is considered "efficient". Moreover, large values of ù are considered "dangerous", while low values of $\dot{u}$ are referred to as "safe". The quantity $\mathbf{u}$ (or fluid acceleration, which also accounts for the convective acceleration, $(\mathbf{u} \cdot \nabla) \mathbf{u}$, is indeed a sensible kinematic quantity to consider, since it is likely to be closely related to fluid pressure, which in turn affects the stress on the retina.

In the present work, we perform systematic experiments on the EVA Phaco-vitrectomy System with different needle sizes and blade shapes to assess its performance.

\section{Methods}

We perform PIV measurements of the flow around the tip of vitreous cutters. Measurements are taken on two vertical planes orthogonal to each other, which are illuminated with a laser sheet. The time resolution of the measurements is such that we manage to resolve the fast oscillations of the flow induced by the high-frequency cutting rate.

We perform all measurements in balanced salt solution (BSS). We also plan to perform experiments in viscoelastic fluid in the near future.

We use cutters with three different diameters $(23,25$, and 27 gauge), two different blade configurations (high-speed cutter [VC] and double cutter [TDC]), and two working modes ("core" and "shaving").

From the PIV measurements, we obtain instantaneous velocity fields, $\mathbf{u}=(u(\mathbf{x}, t)$, $v(\mathbf{x}, t))$. From these instantaneous fields, many other quantities can be obtained. In particular, we derive fluid acceleration, kinetic energy, vorticity, and rate of deformation. Moreover, for each kinematic quantity we perform:

1. spatial averages that give us information about the time evolution of the flow field;

2. time averages that provide a general picture of the averaged spatial distribution of the flow; and

3. time and space averages.

Since the flow is not exactly axisymmetric, it is not possible to obtain an accurate estimate of the volumetric flux from the PIV measurements taken on a plane, which would require a full 3-D reconstruction of the flow field around the tip of the needle. To overcome this difficulty, we employ a different technique. We illuminate a vertical plane of the whole reservoir containing the fluid, and track the position of the free surface in time. Knowing the cross-sectional area of the reservoir, we can evaluate the volumetric flux pumped by the vitreous cutter. We note that, with this approach, we cannot resolve the fast oscillations of the flux related to the cutting frequency. 


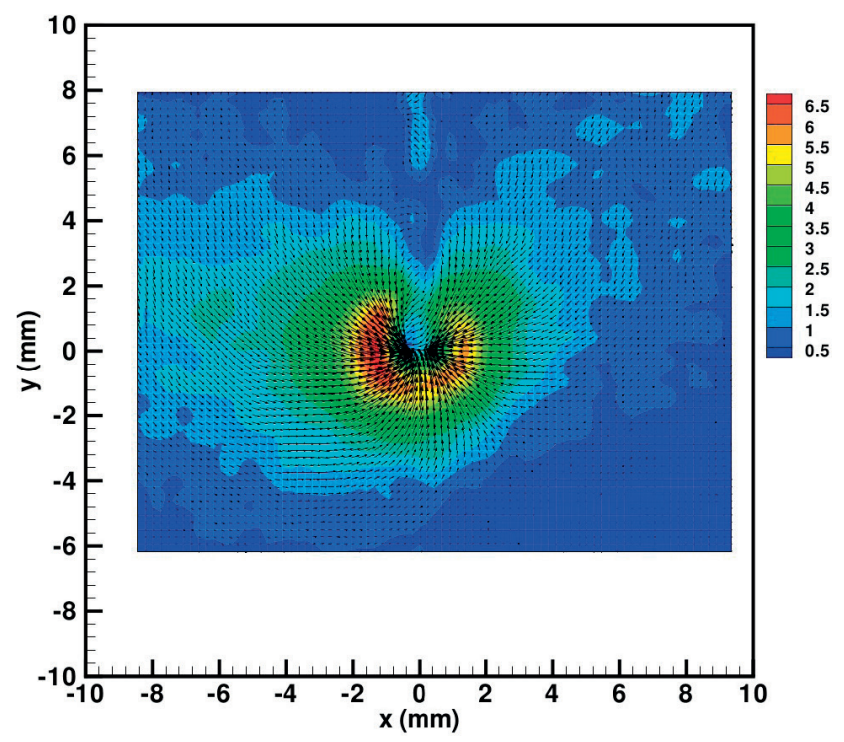

Fig. 1. Example of an instantaneous velocity field (arrows). The color map represents the velocity magnitude in $\mathrm{mm} / \mathrm{s}$.

\section{Results}

In Figure 1 we plot an example of a measured instantaneous velocity field, $\mathbf{u}(\mathbf{x}, t)$, at a given time $t$. This is the basic output of the cross-correlation procedure that is performed to analyze the PIV images. The region depicted in the plot is a square with side $\approx 18 \mathrm{~mm}$, and the quality of the images is such that we manage to compute $\approx 3600$ velocity vectors on the measurement plane. The plane of measurement is not exactly in correspondence to the cutter needle, so that the whole image is occupied by the fluid. The magnitude of the velocity, $|u|$, is also reported in Figure 1 . The velocity field is very regular and approximately symmetrical, as expected on this plane. Note that the slight departure from symmetry can be due to experimental errors, but also to the asymmetry of the suction needle.

In Figure 2 we show examples of the time evolution of the spatially averaged kinetic energy. The spatial average has been taken over a circle centered in correspondence to the cutter cleft with a radius of $3 \mathrm{~mm}$. We note that, obviously, the values of the averaged quantities depend on the size of the region over which averaging is performed. Figure 2 also shows that the kinetic energy has fast oscillations about an average value. These oscillations are due to the cutting cycle, and therefore, have a dominant frequency equal to the cutting one.

In Figure 3 we report a plot that summarizes the main outcomes of the present 


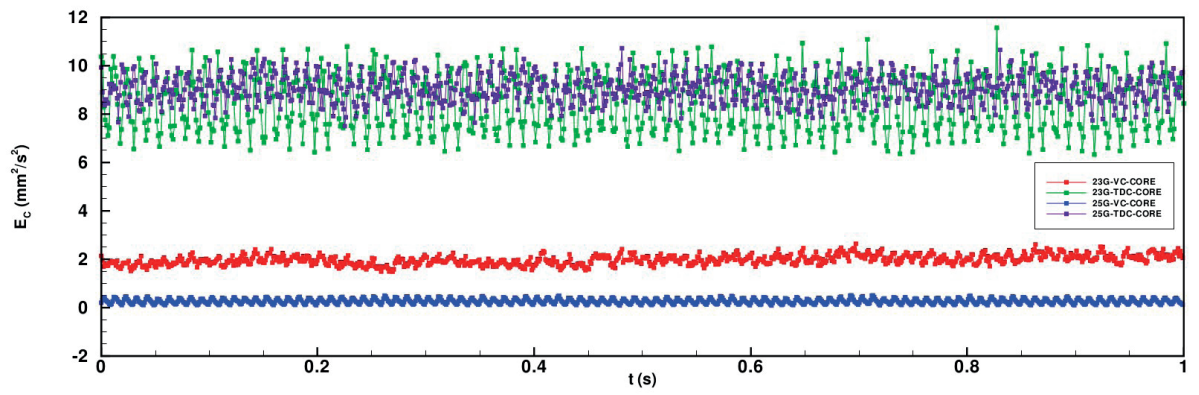

Fig. 2. Example of time evolution of the spatially averaged kinetic energy per unit mass.

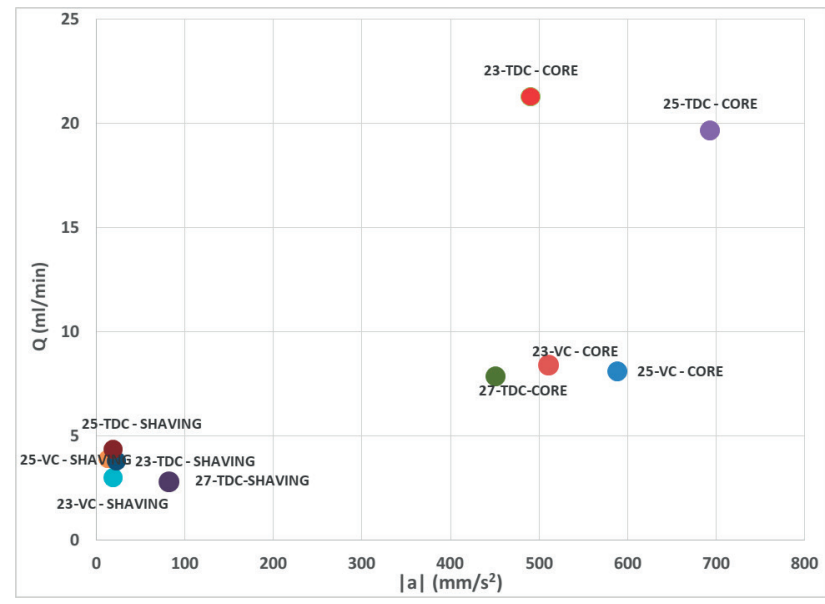

Fig. 3. Diagram showing, for each experiment, flux vs time and space-averaged magnitude of fluid acceleration.

analysis, analogous to Figure 8 in the paper by Rossi et al. ${ }^{1}$ Each point in the plot identifies an experiment. On the $\mathrm{x}$-axis, we report the magnitude of the time- and space-averaged fluid acceleration; on the $y$-axis, the volumetric flux. We note that in each experiment we used the cutter in conditions similar to those adopted in clinical practice.

\section{Conclusion}

The results show that, for a given cutter size and pressure, with the TDC, the velocity, and consequently, the flux, are significantly larger than in the corresponding VC cases. The accelerations, however, have a different behavior. In particular, for the 23-gauge case they are very similar in the TDC and VC cases, in spite of the fact 
that in the former the flux is more than double. For the 25 gauge, the acceleration is greater in the TDC case. However, the difference between the accelerations is much smaller than the differences between the fluxes. These results show that, in the TDC case, the accelerations induced are significantly smaller for a given flux than in the VC case.

\section{Acknowledgements}

This research has been funded by DORC BV, Zuidland, The Netherlands.

\section{References}

1. Rossi T, Querzoli G, Angelini C, et al. Introducing new vitreous cutter blade shapes. Retina. 2014;34:1896-1904. 